\title{
Beneficial effects of exosomes secreted by cardiac-derived progenitor cells and other cell types in myocardial ischemia
}

\author{
Lucio Barile ${ }^{1}$, Giuseppina Milano ${ }^{1,2}$, Giuseppe Vassalli ${ }^{1,2,3}$ \\ ${ }^{1}$ Laboratory of Cellular and Molecular Cardiology, Swiss Institute for Regenerative Medicine (SIRM) and Cardiocentro Ticino Foundation, \\ Lugano, Switzerland; ${ }^{2}$ Heart and Vessel Department, CHUV University of Lausanne Medical Center, Lausanne, Switzerland; ${ }^{3}$ Center for Molecular \\ Cardiology, University of Zürich, Zürich, Switzerland \\ Contributions: (I) Conception and design: L Barile, G Vassalli; (II) Administrative support: None; (III) Provision of study material or patients: L Barile, \\ G Milano; (IV) Collection and assembly of data: L Barile, G Milano; (V) Data analysis and interpretation: All authors; (VI) Manuscript writing: All \\ authors; (VII) Final approval of manuscript: All authors. \\ Correspondence to: Giuseppe Vassalli, MD. Cardiocentro Ticino, via Tesserete 48, 6900 Lugano, Switzerland. \\ Email: giuseppe.vassalli@cardiocentro.org.
}

\begin{abstract}
When injected into acutely infarcted rodent or pig hearts, naturally secreted nanovesicles known as exosomes from cardiac-derived progenitor cells (CPCs) reduce scar size and improve cardiac function. In this regard, exosomes fully mimic the benefits of injecting their parent cells. This recognition paves the way to the development of exosome-based, cell-free treatments for heart disease that could possibly supplant cellbased therapies. Mechanisms of benefit of these vesicles are incompletely understood but cytoprotection, stimulation of angiogenesis, induction of antifibrotic cardiac fibroblasts, and modulation of M1/M2 polarization of macrophages infiltrating the infarcted region can all play important roles. Accordingly, the beneficial molecules carried by CPC-secreted exosomes have been identified only in part but cytoprotective and proangiogenic microRNAs (miRNA) and proteins have been described. Besides CPC-secreted exosomes, vesicles released from other cell types including mesenchymal stem cells (MSCs), embryonic stem cells (ESCs), and induced pluripotent stem cells (iSPCs) have also been associated with cardioprotection. This review aims to discuss recent advances in our understanding of the role of secreted vesicles in cardiac repair, with a focus on CPC-derived exosomes.
\end{abstract}

Keywords: Extracellular vesicles; exosomes; cardiac progenitor cells; myocardial ischemia

Received: 14 August 2017; Accepted: 01 November 2017; Published: 18 November 2017.

doi: $10.21037 /$ sci.2017.11.06

View this article at: http://dx.doi.org/10.21037/sci.2017.11.06

The adult heart hosts a limited number of stem and progenitor cells that exhibit heterogeneous characteristics and fates and have therapeutic potential for cardiac repair and regeneration (1). Multiple methods have been applied to the prospective isolation of putative stem and progenitor cells from heart tissue. Some of these methods rely on the expression of selectable cell surface markers that have been associated with other stem cell populations, hematopoietic bone marrow stem cells in particular, such as c-kit and stem cell antigen-1 (Sca-1) (2,3). Alternate approaches are based on functional properties, such as proliferation and migratory capacity, of cells within heart tissue specimens. When heart tissue explants are cultured ex vivo using the primary tissue culture technique, a cell population migrates out of them within a few days. This population is highly enriched with stem and progenitor cells that exhibit cardiovascular differentiation potential and regenerative activity (4). It therefore looks like stem/progenitor cells preferentially migrate out of ex vivo cultured cardiac explants, proliferate and establish new populations that can be expandend in vitro. These cells express mesenchymal/stromal stem and progenitor cell markers and exhibit mesenchymal multilineage differentiation capability. Thus, they can be viewed as cardiac-resident mesenchymal/stromal stem/ 
progenitor cells. We have used this technique to derive these cells (5), here called cardiac-derived progenitor cells (CPCs). It should be emphasized that these cells are inclusive but not exclusively consistent of cardiac progenitor cells, as functionally defined by cardiac fate specification (6). One other distinction that needs to be preliminarily explained is the one between CPCs and cardiospherederived cells (CDCs). When cultured ex vivo under specific low-adhesion conditions, CPCs spontaneously form spherical aggregates, dubbed cardiospheres, which then give rise to a monolayer of expandable CDCs. Thus, CDCs derive from, but are not identical with, CPCs. Between CPCs and CDCs there is cardiosphere formation, a process associated with cell proliferation and maturational changes including cardiovascular fate commitment as a result of cellcell interactions within these spherical cell aggregates (7). Here, we use the terms CPCs and CDCs according to the original articles from the literature that are reviewed. When general considerations apply to both cell types, however, the term CPCs is also used as inclusive of CDCs, for the sake of simplicity, as will be apparent below.

Intramyocardial injection of autologous CDCs was shown to be safe and potentially beneficial in a phase-I clinical trial in patients after acute myocardial infarction (MI) (8) and phase-II studies are ongoing. Although CDCs can produce new cardiomyocytes, the actual mechanism of benefit in vivo appears to be indirect: injected cells were not found in the heart at late time points after delivery and, following injection of human CDCs in infarcted mouse hearts, 90\% of newly-formed cardiomyocytes and endothelial cells were of endogenous origin (9). In another model, c-kit-positive cardiac progenitors injected into injured hearts generated vanishingly few mature cardiomyocytes but stimulated the proliferation of endogenous cells, which persisted for at least 1 year after injury (10). Thus, exogenous cell delivery seems to enhance endogenous repair via paracrine factors. Current interest in mechanisms of stem cell-mediated cardiac repair therefore is centered on the paracrine hypothesis, which postulates that stem cells secrete beneficial factors that stimulate regeneration and enhance myocardial function. These factors include a wide range of soluble factors released by the conventional and the unconventional secretory pathways, including nanosized membranous extracellular vesicles (EVs) called exosomes, which are released by the unconventional secretory pathway and deliver bioactive molecules to other cells in the heart. Exosome-based mechanisms of stem cell-mediated benefit have recently attracted considerable attention. Glembotski recently phrased this as follows: "The exosomecentric view drives much of the current thinking of those who study of stem cell-mediated repair in the heart" (11).

Initially thought to be small vesicles by which maturating sheep reticulocytes discard obsolete cellular components (12-14), exosomes and other secreted vesicles are now viewed as universal mediators of intercellular communication. Exosomes are nanosized EVs of endosomal origin $(15,16)$. All examined eukaryotic cell types secrete exosomes in culture (17). In vivo, secreted vesicles can be internalized by neighboring cells or circulate in the blood and eventually interact with cells at a distance. EVs carry biologically active molecules such as proteins, lipids, and nucleic acids including mRNA, microRNA (miRNA), long noncoding RNA (lncRNA), and DNA. By delivering nucleic acids to recipient cells, EVs can exchange genetic information between cells (18-21). mRNA carried by mouse exosomes was shown to enter human mast cells in vitro, be translated and produce new mouse proteins in these human cells. Quesenberry et al. (22) reported that EVs derived from either the lung or liver entered bone marrow cells in vitro and induced expression of proteins specific for the originating lung or liver tissue. Moreover, we and others have shown that miRNA delivered via exosomes represses target genes in recipient cells (5,23-26).

This review of the literature summarizes recent advances in our knowledge of the cardioprotective and regenerative activities of exosomes secreted by CPCs (CPC exosomes, for short) and CDCs. In addition, recent data on beneficial effects of exosomes released from other cell types including mesenchymal stem cells (MSCs), embryonic stem cells (ESCs) and induced pluripotent stem cells (iPSCs) in cardiac injury models will be briefly mentioned.

\section{Visualization of exosome release from CPCs}

Using electron transmission microscopy, Sahoo and Losordo (27) described exosome-like vesicles packed in multivesicular bodies of a CPC in a healthy mouse heart and in the cytoplasm of cardiomyocytes from the left ventricle of a healthy human and from a patient with ischemic heart disease. We similarly provided ultrastructural evidence of exosome-like vesicles released by human cardiospheres in vitro and cells in the mouse heart in vivo (28). The vesicles exhibited a characteristic lipid bilayer membrane and were $50-100 \mathrm{~nm}$ in diameter, the size typically associated with exosomes. Fluorescently labeled human CPC exosomes were taken up by HL-1 myxoma atrial cells in vitro (5). 
Subsequent studies addressed the molecular content and functional activity of CPC exosomes.

\section{Benefits of CPC exosomes in myocardial ischemia and ischemia/reperfusion (IR) injury}

In 2010, Lai et al. (29) showed that MSC exosomes injected after myocardial IR injury alleviated myocardial damage. In 2014, we (5) and others [Ibrahim et al. (30)] independently reported on the beneficial effects of exosomes released from human CPCs and CDCs in rodent MI models, respectively. CPCs were derived from right atrial appendage tissue specimens from patients undergoing heart valve surgery (5), while CDCs were obtained from endomyocardial biopsies from the right ventricular aspect of the interventricular septum from healthy hearts or deceased tissue donors (30). Secreted vesicles were purified from CPC and CDC conditioned media by centrifugation at $3,000 \mathrm{~g}$ to remove cellular debris followed by Exoquick ExosomePrecipitation Solution (System Biosciences), as described elsewhere (31). Vesicle populations were heterogeneous in diameter $[\sim 30-160 \mathrm{~nm}$ in the CPC study (5), 15-94 nm in the CDC study (30)] and expressed cell surface (CD63, CD9, CD81) and intracellular (TSG101, Alix) exosome markers. Based on a recent classification by Kowal et al. (16), small ( $<200 \mathrm{~nm})$ particles coenriched in CD63, CD81, CD9, and TSG-101 can be categorized as bone fide exosomes. These vesicles reduced stress-induced cardiomyocyte apoptosis in vitro and stimulated tube formation by human umbilical vein endothelial cells (HUVECs) cultured in Matrigel (Figure 1). By contrast, exosomes released from human normal dermal fibroblasts as a functionally inert cell type in these MI models lacked these bioactivities. When injected into the infarct border zone of rat hearts $60 \mathrm{~min}$ after in vivo coronary artery occlusion, human CPC exosomes improved global heart function (Figure 2), decreased infact size, and increased blood vessel density, as compared with dermal fibroblast exosomes (5). Evidence of benefit of exosomes delivered $60 \mathrm{~min}$ after coronary occlusion is of relevance to potential clinical applications, as a 60 to $120 \mathrm{~min}$ "pain-torevascularization" time can be achieved in part of the patients undergoing a percutaneous coronary intervention because of an acute coronary syndrome. Similar results were reported after intramyocardial injection of human CDC exosomes in immunodeficient mice (30). Pharmacological blockade of exosome secretion by GW4869, which inhibits neutral spingomyelinase 2 [nSMase2; a crucial enzyme in exosome secretion (32)], nullified CDC bioactivity. Exosomes from both CPCs and CDCs were highly enriched with miR-146a, as compared with fibroblast exosomes (5,30). Moreover, miR-146a tissue levels were increased in mouse hearts injected with CDC exosomes. miR-146a knockout (KO) mice exhibited impaired heart function, increased scar mass, decreased infarct wall thickness, and worse post-infarct remodeling compared with wild-type mice of the same strain. Injection of a miR-146a mimic at the time of MI was beneficial in miR-146a KO mice. Furthermore, miR-146a-deficient exosomes produced by transfecting parent CDCs with a miR-146a hairpin inhibitor conferred limited protection to cardiomyocytes against oxidant stress (30). These findings support a cardioprotective role of miR-146a transfer via CDC exosomes.

Using lentiviral knockdown of nSMase2, Lang et al. (32) demonstrated that CDC exosomes contributed to the proangiogenic and promigratory activities of human CDCs in HUVECs while also reducing both apoptosis of cardiomyocytes and proliferation of fibroblasts in vitro. Chen et al. (33) reported that mouse CPC exosomes inhibited cardiomyocyte apoptosis in mouse hearts after IR in vivo. Gray et al. (34) described proangiogenic and antifibrotic activities of mouse CPC exosomes in vitro. Hypoxia stimulated exosome release from CPCs and modified their molecular content, as discussed below. Kervadec et al. (35) showed that CPC-derived EVs recapitulated the beneficial effects of their parent cells in chronic heart failure models.

Gallet et al.(36) recently investigated the benefits of CDC exosomes in a translationally realistic largeanimal model. Pigs subjected to acute MI received human CDC exosomes (or vehicle) by intracoronary or openchest intramyocardial delivery $30 \mathrm{~min}$ after reperfusion. Intramyocardial injection of CDC exosomes decreased infarct size from $80 \% \pm 5 \%$ to $61 \% \pm 12 \%(\mathrm{P}=0.001)$ and preserved left ventricular ejection fraction (LVEF), whereas intracoronary exosome delivery was ineffective. In a randomized placebo-controlled study of convalescent MI, pigs 4 weeks after MI underwent percutaneous intramyocardial delivery of CDC exosomes (or vehicle). Magnetic resonance imaging performed before and 1 month after treatment revealed that exosomes (but not vehicle) preserved LV volumes and LVEF while also decreasing scar size. Histologically, exosomes decreased LV collagen content and cardiomyocyte hypertrophy while also increasing blood vessel density. Thus, CDC exosomes decreased scarring, halted adverse remodeling, and improved LVEF in porcine acute MI and convalescent MI models; however, intramyocardial delivery was required. 


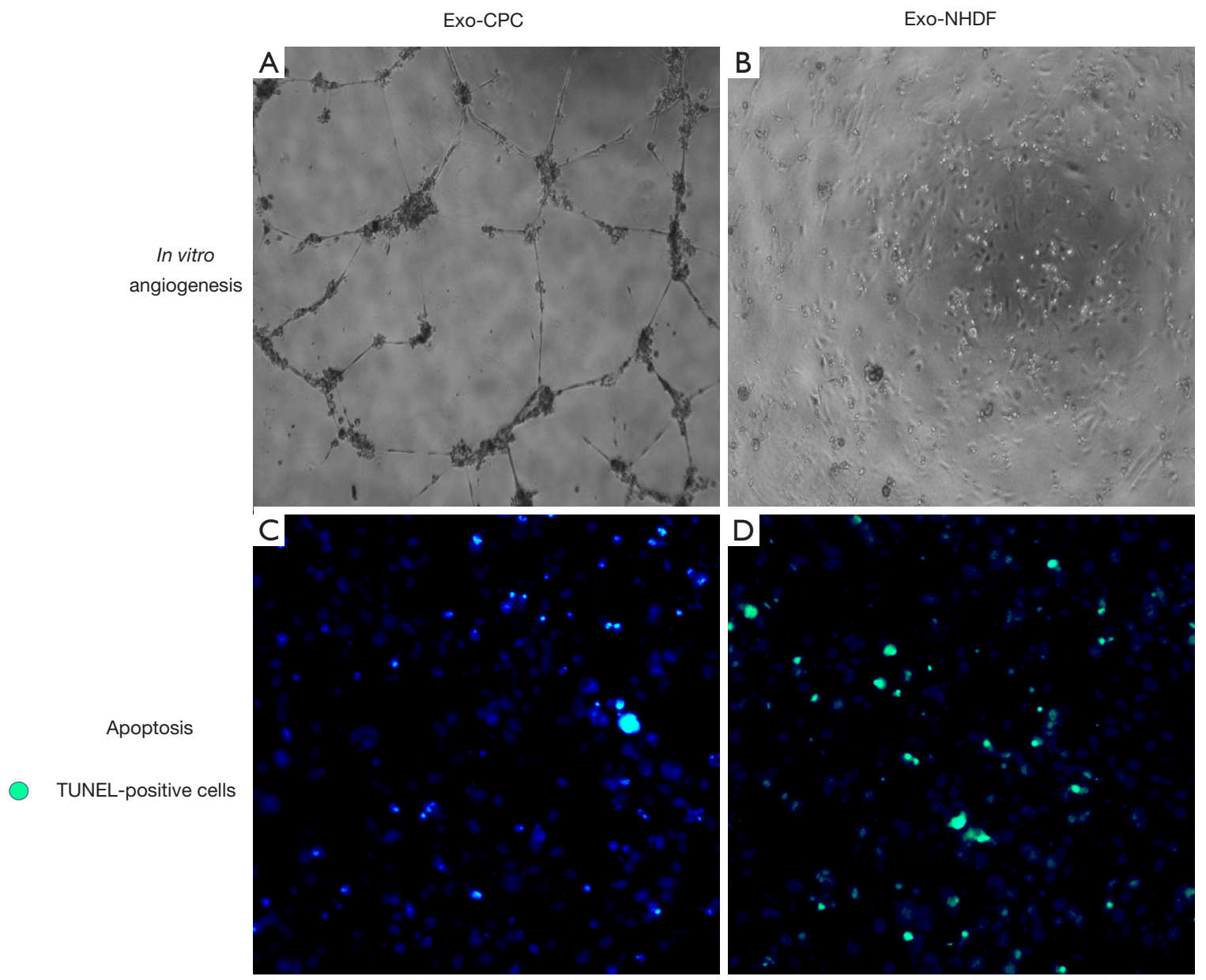

Figure 1 Protective in vitro effects of human CPC exosomes (Exo-CPC), as compared with exosomes from human normal dermal fibroblasts (Exo-NHDF). (A) Formation of vessel-like tubular structures by HUVECs in Matrigel is visible after treatment with Exo-CPC; (B) ExoNHDF lacks such proangiogenic effects; (C) in vitro antiapoptic effects of Exo-CPC in the staurosporine-treated HL-1 cardiomyocytic cell line; (D) Exo-NHDF lacks anti-apoptotic activity. TUNEL-positive cells stain green.

\section{Roles of hypoxia and age on exosome activity}

Hypoxia triggers complex adaptive responses in cardiac cells. Changes in the release and activity of CPC exosomes in response to hypoxia have been addressed recently. In a study by Cosme et al. (37), hypoxia induced significant changes in the secretome, exosome, and whole-cell proteome of cardiac fibroblasts isolated from neonatal mice. In the aforementioned study by Gray et al. (34), hypoxia enhanced exosome release from mouse CPCs while concomitantly altering their molecular content. Exosomes released from hypoxic mouse CPCs showed increased angiogenic activity and decreased ability to upregulate profibrotic genes in fibroblasts stimulated by transforming growth factor $\beta$ (TGF- $\beta$ ). Expression of proangiogenic genes, antifibrotic genes, and a cluster of miRNAs was upregulated in hypoxic CPC-exosomes, which alleviated myocardial fibrosis and improved cardiac function after IR in vivo.

Agarwal et al. (38) elavuated the role of donor age and hypoxia of human pediatric CPC exosomes in a rat model of myocardial IR injury. CPC exosomes derived from neonates improved cardiac function independent of culture oxygen levels, whereas CPC exosomes from older children were not reparative unless subjected to hypoxic conditions. Improvement in cardiac function by hypoxic CPC exosomes from older children was associated with increased angiogenesis, reduced fibrosis, and improved hypertrophy. By contrast, normoxic neonatal CPC exosomes improved 

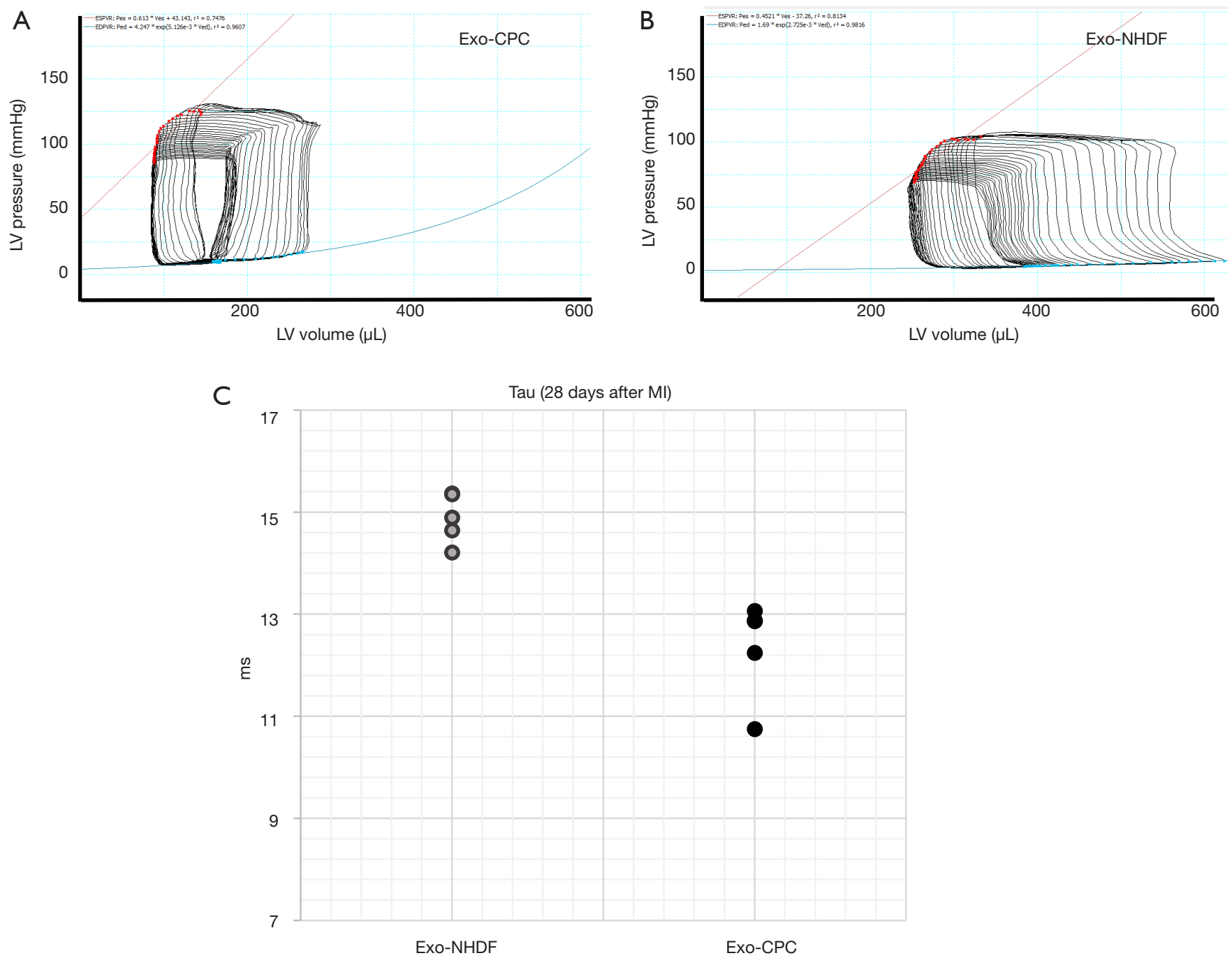

Figure 2 In vivo effects of human CPC exosomes (Exo-CPC), as compared with exosomes from human normal dermal fibroblasts (Exo$\mathrm{NHDF}$ ), on left ventricular (LV) function. Exosomes were injected into infarcted rat hearts in vivo. Four weeks post-MI, LV function was assessed by intraventricular pressure and volume recordings using Millar microcatheters. Representative LV pressure/volume loops, which are shifted to the left in Exo-CPC-treated hearts (A) compared with Exo-NHDF-treated control hearts (B), indicating a decreased ventricular compliance in the latter. From LV pressure/volume loops, the time costant of isovolumic LV pressure fall (Tau), which reflects diastolic relaxation properties was calculated. (C) Tau was reduced in the Exo-CPC group compared with the Exo-NHDF group (n=4 rats/ group) reflecting improved diastolic function.

cardiac function independent of those mechanisms.

Sharma et al. (39) compared functional activities of adult and neonatal CPCs derived from patients aged $>40$ years or $<1$ month, respectively, in an acute MI model. A more robust in vitro proliferative capacity of neonatal CPCs, compared with adult cells, correlated with significantly greater myocardial recovery mediated by neonatal cells in vivo. A single injection of neonatal CPC conditioned medium was more effective than neonatal CPCs, adult CPC-derived conditioned medium, or neonatal CPC exosomes in recovering cardiac function, stimulating neovascularization, and promoting myocardial remodeling. These findings suggest that neonatal CPC-derived conditioned medium may contain beneficial nonexosomal factors. A deep proteomic analysis of the secretome of both neonatal and adult CPC conditioned medium quantified 
the expression pattern of 804 proteins in neonatal CPC conditioned medium and 513 proteins in adult CPC conditioned medium. A literature-based proteomic network analysis identified that 46 and 6 canonical signaling pathways were significantly targeted by neonatal CPC conditioned medium and adult CPC conditioned medium, respectively. Among them, one leading candidate pathway was heat-shock factor-1 (HSF-1). Modulation of HSF-1 by knockdown in neonatal CPCs or overexpression in adult CPCs significantly altered the quality of their secretome. However, this study primarily analyzed CPC conditioned medium, rather than its exosomal fraction.

Collectively, these findings suggest that neonatal and/or hypoxic CPC exosomes may be more protective than adult normoxic exosomes when delivered after acute MI.

\section{Antiapoptotic effects of CPC exosomes}

Although vesicle secretion by CPCs and CDCs is well characterized, the precise target cell type(s) remain(s) undefined. Exosomes from CPCs and CDCs have been shown to exert antiapoptotic effects on cardiomyocytes both in vitro and in vivo $(5,30)$. We reported that human CPC exosomes were enriched in miR-210 and miR-132 compared to dermal fibroblast exosomes, which prevented stress-induced apoptosis of cultured HL-1 cardiomyocytes. HL-1 cells cocultured with CPC exosomes exhibited a time-dependent increase in the intracellular concentrations of miR-210 and miR-132, and a repression of the respective target genes. In line with previous data (40), miR-210 downregulated ephrin A3 and PTP1 (5). The antiapoptotic effect of CDC exosome-delivered miR-146a on cardiomyocytes was mentioned above (30). Exogenous miR-146a was shown to suppress IR injury via targeting of Irak-1 and Traf6 (41), both involved in the Toll-like receptor (TLR) signaling pathway, which may play an important role in the pathology of sterile inflammation, including MI (42). Infarcted hearts injected with CDC exosomes showed increased miR-146a levels, suppression of Irak1 and Traf6, and decreased proinflammatory cytokines (30). miR-146a suppressed NOX-4, which has been linked to oxidative stress and cardiac injury (43), and SMAD4, a member of the TGF- $\beta$ profibrotic pathway (44). Recently, Xiao et al. (45) reported that CPC exosomes prevented cardiomyocyte apoptosis via miR-21 transfer by targeting programmed cell death 4 (PDCD4).

Whether genuine cardiac regeneration occurs has been not definitively proven. When injected 21 days after MI in mice, a time point at which myocardial scar is well established, CDC exosomes were still associated with decreases in cardiomyocyte apoptosis and scar size, and increases in viable mass, microvessels density and cardiac function (30). These findings support genuine regeneration. Additional mechanisms of benefit of exosomes include proangiogenic effects and changes in the secretory profile of fibroblasts as well as M1/M2 macrophage polarization.

\section{Proangiogenic activities of CPC exosomes}

We and others have demonstrated proangiogenic activities of CPC exosomes both in vitro and in vivo $(5,30)$, as mentioned above. Compared with dermal fibroblast exosomes, human CPC exosomes were enriched in miR-210 and miR-132, which play important roles in angiogenesis and vascular remodeling $(40,46)$. We and others reported miR-132mediated RasGap-p120 downregulation and increased tube formation in HUVECs $(5,46)$. Vrijsen et al. (47) reported that CPC exosomes stimulated the migration of endothelial cells via their matrix metalloproteinase (MMP) content.

Besides EVs derived from CPCs and CDCs, proangiogenic effects of exosomes secreted by $\mathrm{CD} 34^{+}$stem cells have been investigated. These cells have shown promising results in clinical cell therapy trials of therapeutic angiogenesis in patients with critical limb ischemia (48), refractory angina pectoris (49), or left ventricular dysfunction post-MI (50). Sahoo et al. (27) reported that exosomes released from human $\mathrm{CD} 34^{+}$stem cells mediated their proangiogenic paracrine activity. When injected into mouse ischemic hindlimb tissue, these exosomes, but not $\mathrm{CD}_{3} 4^{+}$stem cell conditioned medium depleted of its exosomal fraction, mimicked the beneficial activity of parent $\mathrm{CD} 34^{+}$stem cells by improving ischemic limb perfusion, capillary density, motor function, and limb amputation rates (51). Exosomes from $\mathrm{CD} 34^{+}$stem cells were enriched with proangiogenic miRNAs including miR126-3p. Knocking down miR-126-3p from these exosomes abolished their angiogenic activity both in vitro and in vivo. miR-126-3p was shown to enhance angiogenesis by suppressing the expression of its known target, SPRED1, simultaneously modulating the expression of genes involved in angiogenic pathways such as vascular endothelial growth factor (VEGF), angiopoietin 1, matrix metallopeptidase 9, and thrombospondin 1 in endothelial cells. Proangiogenic activities of exosomes secreted by endothelial cells have also been described $(52,53)$. 
Collectively, these findings demonstrate significant proangiogenic activities of exosomes released from CPCs, CDCs, $\mathrm{CD}_{3} 4^{+}$stem cells, and endothelial cells.

\section{Antifibrotic activities of CDC exosomes}

Besides CPCs, cardiomyocytes and endothelial cells, fibroblasts play an important role in cardiac repair. Tseliou et al. (54) reported that CDC exosomes altered the secretory profile of dermal fibroblasts, rendering them antifibrotic, antiapoptotic, and proangiogenic. CDC exosome priming induced the secretion of markedly higher levels of stromal cell-derived factor-1 (SDF-1) and VEGF by fibroblasts while also dramatically changing miRNA profiles of their secreted vesicles. Intramyocardial injection of CDC exosome-primed fibroblasts, unlike unprimed fibroblasts, improved ventricular function and vessel density while also reducing scar mass in rat hearts after chronic MI. These results were viewed as evidence that CDC exosomes may switch inert fibroblasts to cells that contribute to cardiac repair. Most recently, Wang et al. (55) reported that macrophage-derived exosomes containing mir-155 suppressed fibroblast proliferation and promoted fibroblast inflammation during cardiac injury. Collectively, these findings suggest that exosomes may modify cardiac repair via modulation of fibroblast function.

\section{Macrophage polarization by CDC exosomes}

Early after acute MI, macrophages migrate into the infarcted region and participate in myocardial healing and inflammation. The balance of M1 vs. M2 polarized macrophages has been shown to influence cardiac repair (56-58). Using rat and pig IR injury models, de Couto et al. (59) showed that intracoronary infusion of CDC exosomes, but not inert fibroblast exosomes, reduced the number of $\mathrm{CD} 68^{+}$macrophages within infarcted tissue and modified the polarization state of macrophages. These changes were associated with cellular postconditioning. Expression of proinflammatory genes Nos 2 and Tnf was significantly attenuated in cardiac macrophages isolated from CDC exosome-treated animals. In vitro, rat bone marrow-derived macrophages exposed to CDC exosomes exhibited a distinctive shift in macrophage polarization (not observed with fibrolast exosomes), including increased antiinflammatory gene expression levels (Arg1, IL $4 r a$, $T g f b 1$ and $V e g f a$, but not Tnf). Reverse pathway analysis of whole-transcriptome data from CDC exosome-primed macrophages implicated miR-181b as a significant candidate mediator of CDC-induced macrophage polarization, and protein kinase $\mathrm{C} \delta(\mathrm{PKC} \delta)$ as a downstream target. Otherwise inert fibroblast exosomes loaded selectively with miR-181b altered macrophage phenotype and conferred cardioprotective efficacy in a rat MI model. Adoptive transfer of PKC $\delta$-suppressed macrophages recapitulated cardioprotection.

Pig bone marrow-derived macrophages exposed to CDC exosomes exhibited a similar polarization shift. Thus, CDC exsosomes recapitulated the effects of CDC therapy on cardiac macrophages within infarcted hearts (60-62). Cambier et al. (63) recently reported that the most abundant RNA species in CDC vesicles was a $\mathrm{Y}$ RNA fragment (YF1) and that its relative abundance in the vesicles correlated with CDC potency in vivo. The vesicles actively transferred fluorescently labeled YF1 from CDCs to macrophages. Direct transfection of macrophages with YF1 induced transcription and secretion of IL-10. YF1-primed macrophages cocultured with rat cardiomyocytes protected them against oxidative stress through induction of IL-10. In vivo, intracoronary injection of vesicle YF1 after IR reduced infarct size. A fragment of Y RNA, highly enriched in CDC vesicles, was found to alter $I l 10$ gene expression and increase IL-10 protein secretion. Collectively, these findings suggest that CDC-derived vesicles may modulate macrophage polarization and YF1 may mediate this effect.

\section{Exosomes mediate remote ischemic preconditioning}

Remote ischemic preconditioning of the heart is induced by brief ischemic insults inflicted on a remote organ or a remote area of the heart before a sustained cardiac ischemia (64). It has been shown that cardioprotection by remote ischemic preconditioning of the rat heart is mediated by extracellular vesicles including exosomes. In a study by Giricz et al. (65), coronary perfusates of isolated-perfused rat hearts exposed to $3 \times 5-5$ min global ischemia and reperfusion, or coronary perfusates depleted of vesicles, were given to another set of recipient isolated hearts. Infarct size was determined after $30 \mathrm{~min}$ global ischemia and $120 \mathrm{~min}$ reperfusion. Ischemic preconditioning markedly increased vesicle release from the heart. Administration of coronary perfusate from ischemic preconditioning donor hearts reduced infarct size in non-preconditioned recipient hearts, similarly to cardioprotection mediated by ischemic preconditioning 
on the donor hearts. Vesicle depletion abolished cardioprotection in recipient hearts. Thus, vesicles released from the heart after ischemic preconditioning were required for cardioprotection by remote ischemic preconditioning.

Yamaguchi et al. (66) reported that remote ischemic conditioning alleviated left ventricular remodeling and interstitial fibrosis in the boundary region after MI via exosomes. The protective effect was associated with increased expression of miR-29a, a key regulator of tissue fibrosis, in exosomes and the marginal area of MI. Insulinlike growth factor-1 (IGF-1) receptor, which signals in the cardioprotective IGF-1 signaling pathway, was highly expressed both in the exosomes and remote noninfarcted myocardium after remote ischemic conditioning.

Li et al. (67) reported that remote ischemic preconditioning, to the opposite of IR injury, increased miR-144 levels in mouse myocardium and miR-144 precursor in exosomes. Systemic treatment with miR-144 increased P-Akt, P-GSK3 $\beta$ and P-p44/42 MAPK, and induced early and delayed cardioprotection resulting in improved functional recovery and decreased infarct size similar to that achieved by remote ischemic preconditioning. Conversely, systemic administration of a specific antisense oligonucleotide reduced myocardial levels of miR-144 and abrogated cardioprotection by remote ischemic preconditioning. These results support a role for miR-144 and a possible contribution of miR-144 transported by exosomes in cardioprotection induced by remote ischemic preconditioning.

\section{MSC-mediated paracrine signaling}

Cardioprotective activities of MSC exosomes (29) have been mentioned above. Mayourian et al. (68) recently addressed the relative effects of human MSC-mediated paracrine signaling and heterocellular coupling on human cardiac contractility and arrhythmogenicity by integrating experimental and computational approaches. Excitationcontraction simulations of MSC paracrine signaling only and combined heterocellular coupling and paracrine signaling on human cardiomyocytes were representative of human engineered cardiac tissue contractile function measurements. MSC-mediated effects were most pronounced under paracrine signaling-only conditions, where developed force increased approximately 4-fold compared to nonMSC-supplemented controls during physiologic $1-\mathrm{Hz}$ pacing. Simulations predicted contractility of isolated healthy and ischemic adult human cardiomyocytes would be minimally sensitive to MSC-mediated heterocellular coupling, driven primarily by paracrine signaling. Treating human engineered cardiac tissue with exosomes-enriched, but not exosomes-depleted, fractions of the human MSC secretome recapitulated the effects observed with human MSC conditioned media on human engineered cardiac tissue developed force and expression of calcium handling genes (e.g., SERCA2a, L-type calcium channel). These results provide novel insights into the role of exosomes in human MSC paracrine-mediated effects on contractility.

\section{Cardioprotection by ESC-derived exosomes}

Khan et al. (69) addressed the effect of mouse ESC-derived exosomes for the repair of ischemic myocardium and whether c-kit ${ }^{+} \mathrm{CPC}$ function can be enhanced with these vesicles. ESC exosomes enhanced neovascularization, CPC survival, proliferation and cardiac commitment, along with an increase in cardiac function and a decrease in fibrosis post-MI. The number of $\mathrm{c}-\mathrm{kit}^{+} \mathrm{CPCs}$ was increased 8 weeks after in vivo ESC exosome transfer, and formation of bona fide new cardiomyocytes in the ischemic heart was reported. miRNA arrays revealed significant enrichment of miR290-295 cluster and particularly miR-294 in ESC exosomes. The investigators linked the beneficial effect of ESC exosomes to delivery of ESC-specific miR-294 to CPCs promoting survival, cell cycle progression, and proliferation. The regenerative ability of ESC exosomes has not been directly compared to that of vesicles derived from adult cells.

\section{Cardioprotection by exosomes from iPSC}

iPSC culture medium was shown to improve alveolar epithelial wound repair in a lung epithelial wound-healing model and reduce lung fibrosis in vivo (70). The therapeutic potential of exosomes from iPSCs and their derivatives for treatment of heart disease has been discussed recently (71). Wang et al. (72) reported that iPSC exosomes protected cardiomyocytes against $\mathrm{H}_{2} \mathrm{O}_{2}$-induced oxidative stress by inhibiting caspase $3 / 7$ activation and attenuated myocardial IR injury in mice via delivery of cardioprotective miRNAs including miR-21 and miR-210. Bobis-Wozowicz et al. (73) showed that microvesicles from hiPSCs were able to deliver bioactive molecules, including mRNA, miRNA, and proteins to human cardiac mesenchymal stromal cells and exert protective effects by affecting their transcriptomes and proteomic profiles, and by promoting cardiac and endothelial differentiation of these cells. Besides iPSC exosomes, exosomes secreted by iPSC-derived MSCs have 
been investigated. The latter improved local angiogenesis in a limb ischemia mouse model and attenuated hepatic IR injury possibly via anti-inflammatory, anti-oxidant, and anti-apoptotic activities $(74,75)$. These findings exemplify the therapeutic potential of iPSC exosomes and EVs from iPSC-derived MSCs.

\section{Cardioprotection by exosomes from iPSC-derived cardiomyocytes}

The ability of ESC-derived and iPSC-derived cardiomyocytes to mitigate ischemic myocardial injury and improve cardiac function after MI has been demonstrated in animal models $(3,76)$. This approach uses autologous cells, thus avoiding alloimmune rejection. iPSC-derived cardiomyocytes from human placenta amniotic MSCs engrafted successfully in immunocompetent SVJ mice and improved myocardial viability and cardiac function after MI $(77,78)$. These exosomes transported several upregulated miRNAs, including miR-92a which has been associated with antiapoptotic, proangiogenic, and antifibrotic effects. Lee et al. (79) compared ESC-derived cardiomyocytes and iPSC-derived cardiomyocytes with respect to their ability to restore cardiac function in a rat MI model, as well as their exosomal secretome. Both cell types injected into nude rats post-MI comparably improved cardiac function. Both groups contained an identical repertoire of miRNAs and lncRNAs, including some that are known to be cardioprotective. Thus, exosomes from iPSCderived cardiomyocytes could replace those from ESCderived cardiomyocytes in therapeutic applications, making autologous approaches possible.

\section{Pathophysiological mechanisms involving exosomes}

It should be pointed out that exosomes may participate in both beneficial and pathophysiological processes, depending on their cells of origin and the functional status of these cells. For example, Halkein et al. (80) showed that miR146a-rich exosomes secreted by endothelial cells were taken up by cardiomyocytes and induced contractile dysfunction in a post-partum cardiomyopathy model. As mentioned above, miR-146a transferred via CPC exosomes has been tied to cardioprotection (30). Thus, an individual miRNA transferred via exosomes from different parent cells and/ or under different environmental conditions may elicit divergent biological responses. Bang et al. (81) reported that cardiac fibroblast-derived miRNA passenger strandenriched exosomes were able to mediate cardiomyocyte hypertrophy. Jiang et al. (82) showed that exosomes purified from serum of pediatric dilated cardiomyopathy patients induced pathological changes in gene expression in neonatal rat cardiomyocytes and human iPSC-derived cardiomyocytes with similar directionality as in diseased hearts, and that serum of such patients induced pathological hypertrophy in cells. Finally, Wang et al. (83) reported that exosomes secreted by cardiomyocytes from adult Goto-Kakizaki rats, a commonly used animal model of type 2 diabetes, inhibited proliferation, migration, and tube-formation by mouse cardiac endothelial cells in a miR-320-dependent manner while exosomes secreted by cardiomyocytes from control Wistar rats induced opposite effects. More studies of the contributions of exosomes to cardiovascular diseases are warranted.

\section{Concluding remarks}

Collectively, accumulating experimental evidence indicates beneficial effects of exosomes released from CPCs and other cell types in ischemic heart disease. These exosomes essentially recapitulate the therapeutic bioactivities of their cells of origin suggesting that vesicle-based, cellfree treatments have a potential for supplanting cellbased therapies in the near future. Concomitantly, pathophysiological roles of exosomes in cardiovascular disease also need to be investigated.

\section{Acknowledgements}

This work was supported in part by Swiss National Science Foundation 310030_169194 grant and a Stiftung für Herzund Kreislaufkrankheiten (Hergiswil, Switzerland) grant to G Vassalli.

\section{Footnote}

Conflicts of Interest: The authors have no conflicts of interest to declare.

\section{References}

1. Gerbin KA, Murry CE. The winding road to regenerating the human heart. Cardiovasc Pathol 2015;24:133-40.

2. Barile L, Messina E, Giacomello A, et al. Endogenous cardiac stem cells. Prog Cardiovasc Dis 2007;50:31-48. 
3. Laflamme MA, Murry CE. Heart regeneration. Nature 2011;473:326-35.

4. Messina E, De Angelis L, Frati G, et al. Isolation and expansion of adult cardiac stem cells from human and murine heart. Circ Res 2004;95:911-21.

5. Barile L, Lionetti V, Cervio E, et al. Extracellular vesicles from human cardiac progenitor cells inhibit cardiomyocyte apoptosis and improve cardiac function after myocardial infarction. Cardiovasc Res 2014;103:530-41.

6. Bondue A, Blanpain C. Mesp1: a key regulator of cardiovascular lineage commitment. Circ Res 2010;107:1414-27.

7. Barile L, Gherghiceanu M, Popescu LM, et al. Human cardiospheres as a source of multipotent stem and progenitor cells. Stem Cells Int 2013;2013:916837.

8. Makkar RR, Smith RR, Cheng K, et al. Intracoronary cardiosphere-derived cells for heart regeneration after myocardial infarction (CADUCEUS): a prospective, randomised phase 1 trial. Lancet 2012;379:895-904.

9. Chimenti I, Smith RR, Li TS, et al. Relative roles of direct regeneration versus paracrine effects of human cardiosphere-derived cells transplanted into infarcted mice. Circ Res 2010;106:971-80.

10. Tang XL, Li Q, Rokosh G, et al. Long-Term Outcome of Administration of c-kit(POS) Cardiac Progenitor Cells After Acute Myocardial Infarction: Transplanted Cells Do not Become Cardiomyocytes, but Structural and Functional Improvement and Proliferation of Endogenous Cells Persist for at Least One Year. Circ Res 2016;118:1091-105.

11. Glembotski CC. Expanding the Paracrine Hypothesis of Stem Cell-Mediated Repair in the Heart: When the Unconventional Becomes Conventional. Circ Res 2017;120:772-4.

12. Trams EG, Lauter CJ, Salem N, Jr., et al. Exfoliation of membrane ecto-enzymes in the form of micro-vesicles. Biochim Biophys Acta 1981;645:63-70.

13. Pan BT, Johnstone RM. Fate of the transferrin receptor during maturation of sheep reticulocytes in vitro: selective externalization of the receptor. Cell 1983;33:967-78.

14. Johnstone RM. Revisiting the road to the discovery of exosomes. Blood Cells Mol Dis 2005;34:214-9.

15. Simons M, Raposo G. Exosomes--vesicular carriers for intercellular communication. Curr Opin Cell Biol 2009;21:575-81.

16. Kowal J, Arras G, Colombo M, et al. Proteomic comparison defines novel markers to characterize heterogeneous populations of extracellular vesicle subtypes. Proc Natl Acad Sci U S A 2016;113:E968-77.

17. Ibrahim A, Marban E. Exosomes: Fundamental Biology and Roles in Cardiovascular Physiology. Annu Rev Physiol 2016;78:67-83.

18. Mittelbrunn M, Sanchez-Madrid F. Intercellular communication: diverse structures for exchange of genetic information. Nat Rev Mol Cell Biol 2012;13:328-35.

19. Deregibus MC, Cantaluppi V, Calogero R, et al. Endothelial progenitor cell derived microvesicles activate an angiogenic program in endothelial cells by a horizontal transfer of mRNA. Blood 2007;110:2440-8.

20. Skog J, Wurdinger T, van Rijn S, et al. Glioblastoma microvesicles transport RNA and proteins that promote tumour growth and provide diagnostic biomarkers. Nat Cell Biol 2008;10:1470-6.

21. Mizrak A, Bolukbasi MF, Ozdener GB, et al. Genetically engineered microvesicles carrying suicide mRNA/ protein inhibit schwannoma tumor growth. Mol Ther 2013;21:101-8.

22. Quesenberry PJ, Aliotta JM. Cellular phenotype switching and microvesicles. Adv Drug Deliv Rev 2010;62:1141-8.

23. Valadi H, Ekstrom K, Bossios A, et al. Exosome-mediated transfer of mRNAs and microRNAs is a novel mechanism of genetic exchange between cells. Nat Cell Biol 2007;9:654-9.

24. Montecalvo A, Larregina AT, Shufesky WJ, et al. Mechanism of transfer of functional microRNAs between mouse dendritic cells via exosomes. Blood 2012;119:756-66.

25. Pegtel DM, Cosmopoulos K, Thorley-Lawson DA, et al. Functional delivery of viral miRNAs via exosomes. Proc Natl Acad Sci U S A 2010;107:6328-33.

26. Squadrito ML, Baer C, Burdet F, et al. Endogenous RNAs modulate microRNA sorting to exosomes and transfer to acceptor cells. Cell Rep 2014;8:1432-46.

27. Sahoo S, Klychko E, Thorne T, et al. Exosomes from human CD34(+) stem cells mediate their proangiogenic paracrine activity. Circ Res 2011;109:724-8.

28. Barile L, Gherghiceanu M, Popescu LM, et al. Ultrastructural evidence of exosome secretion by progenitor cells in adult mouse myocardium and adult human cardiospheres. J Biomed Biotechnol 2012;2012:354605.

29. Lai RC, Arslan F, Lee MM, et al. Exosome secreted by MSC reduces myocardial ischemia/reperfusion injury. Stem Cell Res 2010;4:214-22.

30. Ibrahim AG, Cheng K, Marban E. Exosomes as critical agents of cardiac regeneration triggered by cell therapy. 
Stem Cell Reports 2014;2:606-19.

31. Taylor DD, Zacharias W, Gercel-Taylor C. Exosome isolation for proteomic analyses and RNA profiling. Methods Mol Biol 2011;728:235-46.

32. Lang JK, Young RF, Ashraf H, et al. Inhibiting Extracellular Vesicle Release from Human Cardiosphere Derived Cells with Lentiviral Knockdown of nSMase2 Differentially Effects Proliferation and Apoptosis in Cardiomyocytes, Fibroblasts and Endothelial Cells In Vitro. PLoS One 2016;11:e0165926.

33. Chen L, Wang Y, Pan Y, et al. Cardiac progenitorderived exosomes protect ischemic myocardium from acute ischemia/reperfusion injury. Biochem Biophys Res Commun;431:566-71.

34. Gray WD, French KM, Ghosh-Choudhary S, et al. Identification of therapeutic covariant microRNA clusters in hypoxia-treated cardiac progenitor cell exosomes using systems biology. Circ Res 2015;116:255-63.

35. Kervadec A, Bellamy V, El Harane N, et al. Cardiovascular progenitor-derived extracellular vesicles recapitulate the beneficial effects of their parent cells in the treatment of chronic heart failure. J Heart Lung Transplant 2016;35:795-807.

36. Ghaleh R, Dawkins JF, Valle J, et al. Exosomes secreted by cardiosphere-derived cells reduce scarring, attenuate adverse remodeling and improve function in acute and chronic porcine myocardial infarction. European Heart Journal 2017;38:201-11.

37. Cosme J, Guo H, Hadipour-Lakmehsari S, et al. HypoxiaInduced Changes in the Fibroblast Secretome, Exosome, and Whole-Cell Proteome Using Cultured, CardiacDerived Cells Isolated from Neonatal Mice. J Proteome Res 2017;16:2836-47.

38. Agarwal U, George A, Bhutani S, et al. Experimental, Systems, and Computational Approaches to Understanding the MicroRNA-Mediated Reparative Potential of Cardiac Progenitor Cell-Derived Exosomes From Pediatric Patients. Circ Res 2017;120:701-12.

39. Sharma S, Mishra R, Bigham GE, et al. A Deep Proteome Analysis Identifies the Complete Secretome as the Functional Unit of Human Cardiac Progenitor Cells. Circ Res 2017;120:816-34.

40. Hu S, Huang M, Li Z, et al. MicroRNA-210 as a novel therapy for treatment of ischemic heart disease. Circulation 2010;122:S124-31.

41. Cheng K, Malliaras K, Smith RR, et al. Human cardiosphere-derived cells from advanced heart failure patients exhibit augmented functional potency in myocardial repair. JACC Heart Fail 2014;2:49-61.

42. Arslan F, Lai RC, Smeets MB, et al. Mesenchymal stem cell-derived exosomes increase ATP levels, decrease oxidative stress and activate PI3K/Akt pathway to enhance myocardial viability and prevent adverse remodeling after myocardial ischemia/reperfusion injury. Stem Cell Res 2013;10:301-12.

43. Infanger DW, Cao X, Butler SD, et al. Silencing nox4 in the paraventricular nucleus improves myocardial infarction-induced cardiac dysfunction by attenuating sympathoexcitation and periinfarct apoptosis. Circ Res 2010;106:1763-74.

44. Liu Z, Lu CL, Cui LP, et al. MicroRNA-146a modulates TGF-beta1-induced phenotypic differentiation in human dermal fibroblasts by targeting SMAD4. Arch Dermatol Res 2012;304:195-202.

45. Xiao J, Pan Y, Li XH, et al. Cardiac progenitor cellderived exosomes prevent cardiomyocytes apoptosis through exosomal miR-21 by targeting PDCD4. Cell Death Dis 2016;7:e2277.

46. Katare R, Riu F, Mitchell K, et al. Transplantation of human pericyte progenitor cells improves the repair of infarcted heart through activation of an angiogenic program involving micro-RNA-132. Circ Res 2011;109:894-906

47. Vrijsen KR, Maring JA, Chamuleau SA, et al. Exosomes from Cardiomyocyte Progenitor Cells and Mesenchymal Stem Cells Stimulate Angiogenesis Via EMMPRIN. Adv Healthc Mater 2016;5:2555-65.

48. Losordo DW, Kibbe MR, Mendelsohn F, et al. A randomized, controlled pilot study of autologous CD34+ cell therapy for critical limb ischemia. Circ Cardiovasc Interv 2012;5:821-30.

49. Povsic TJ, Henry TD, Traverse JH, et al. The RENEW Trial: Efficacy and Safety of Intramyocardial Autologous CD34(+) Cell Administration in Patients With Refractory Angina. JACC Cardiovasc Interv 2016;9:1576-85.

50. Quyyumi AA, Vasquez A, Kereiakes DJ, et al. PreSERVEAMI: A Randomized, Double-Blind, Placebo-Controlled Clinical Trial of Intracoronary Administration of Autologous CD34+ Cells in Patients With Left Ventricular Dysfunction Post STEMI. Circ Res 2017;120:324-31.

51. Mathiyalagan P, Liang Y, Kim D, et al. Angiogenic Mechanisms of Human CD34+ Stem Cell Exosomes in the Repair of Ischemic Hindlimb. Circ Res 2017;120:1466-76.

52. de Jong OG, Verhaar MC, Chen Y, et al. Cellular stress conditions are reflected in the protein and RNA content of endothelial cell-derived exosomes. J Extracell Vesicles 
2012;1.

53. van Balkom BW, de Jong OG, Smits M, et al. Endothelial cells require miR-214 to secrete exosomes that suppress senescence and induce angiogenesis in human and mouse endothelial cells. Blood 2013;121:3997-4006, S1-15.

54. Tseliou E, Fouad J, Reich H, et al. Fibroblasts Rendered Antifibrotic, Antiapoptotic, and Angiogenic by Priming With Cardiosphere-Derived Extracellular Membrane Vesicles. J Am Coll Cardiol 2015;66:599-611.

55. Wang C, Zhang C, Liu L, et al. Macrophage-Derived mir-155-Containing Exosomes Suppress Fibroblast Proliferation and Promote Fibroblast Inflammation during Cardiac Injury. Mol Ther 2017;25:192-204.

56. Courties $G$, Heidt T, Sebas $M$, et al. In vivo silencing of the transcription factor IRF5 reprograms the macrophage phenotype and improves infarct healing. J Am Coll Cardiol 2014;63:1556-66.

57. Weirather J, Hofmann UD, Beyersdorf N, et al. Foxp3+ CD4+ T cells improve healing after myocardial infarction by modulating monocyte/macrophage differentiation. Circ Res 2014;115:55-67.

58. Ben-Mordechai T, Palevski D, Glucksam-Galnoy Y, et al. Targeting macrophage subsets for infarct repair. J Cardiovasc Pharmacol Ther 2015;20:36-51.

59. de Couto G, Gallet R, Cambier L, et al. Exosomal MicroRNA Transfer Into Macrophages Mediates Cellular Postconditioning. Circulation 2017;136:200-14.

60. de Couto G, Liu W, Tseliou E, et al. Macrophages mediate cardioprotective cellular postconditioning in acute myocardial infarction. J Clin Invest 2015;125:3147-62.

61. Kanazawa H, Tseliou E, Malliaras K, et al. Cellular postconditioning: allogeneic cardiosphere-derived cells reduce infarct size and attenuate microvascular obstruction when administered after reperfusion in pigs with acute myocardial infarction. Circ Heart Fail 2015;8:322-32.

62. Kanazawa H, Tseliou E, Dawkins JF, et al. Durable Benefits of Cellular Postconditioning: Long-Term Effects of Allogeneic Cardiosphere-Derived Cells Infused After Reperfusion in Pigs with Acute Myocardial Infarction. J Am Heart Assoc 2016;5:e002796.

63. Cambier L, de Couto G, Ibrahim A, et al. Y RNA fragment in extracellular vesicles confers cardioprotection via modulation of IL-10 expression and secretion. EMBO Mol Med 2017;9:337-52.

64. Heusch G, Botker HE, Przyklenk K, et al. Remote ischemic conditioning. J Am Coll Cardiol 2015;65:177-95.

65. Giricz Z, Varga ZV, Baranyai T, et al. Cardioprotection by remote ischemic preconditioning of the rat heart is mediated by extracellular vesicles. J Mol Cell Cardiol 2014;68:75-8.

66. Yamaguchi T, Izumi Y, Nakamura Y, et al. Repeated remote ischemic conditioning attenuates left ventricular remodeling via exosome-mediated intercellular communication on chronic heart failure after myocardial infarction. Int J Cardiol 2015;178:239-46.

67. Li J, Rohailla S, Gelber N, et al. MicroRNA-144 is a circulating effector of remote ischemic preconditioning. Basic Res Cardiol 2014;109:423.

68. Mayourian J, Cashman TJ, Ceholski DK, et al. Experimental and Computational Insight Into Human Mesenchymal Stem Cell Paracrine Signaling and Heterocellular Coupling Effects on Cardiac Contractility and Arrhythmogenicity. Circ Res 2017;121:411-23.

69. Khan M, Nickoloff E, Abramova T, et al. Embryonic stem cell-derived exosomes promote endogenous repair mechanisms and enhance cardiac function following myocardial infarction. Circ Res 2015;117:52-64.

70. Gazdhar A, Grad I, Tamo L, et al. The secretome of induced pluripotent stem cells reduces lung fibrosis in part by hepatocyte growth factor. Stem Cell Res Ther 2014;5:123.

71. Jung JH, Fu X, Yang PC. Exosomes Generated From iPSC-Derivatives: New Direction for Stem Cell Therapy in Human Heart Diseases. Circ Res 2017;120:407-17.

72. Wang Y, Zhang L, Li Y, et al. Exosomes/microvesicles from induced pluripotent stem cells deliver cardioprotective miRNAs and prevent cardiomyocyte apoptosis in the ischemic myocardium. Int J Cardiol 2015;192:61-9.

73. Bobis-Wozowicz S, Kmiotek K, Sekula M, et al. Human Induced Pluripotent Stem Cell-Derived Microvesicles Transmit RNAs and Proteins to Recipient Mature Heart Cells Modulating Cell Fate and Behavior. Stem Cells 2015;33:2748-61.

74. Hu GW, Li Q, Niu X, et al. Exosomes secreted by humaninduced pluripotent stem cell-derived mesenchymal stem cells attenuate limb ischemia by promoting angiogenesis in mice. Stem Cell Res Ther 2015;6:10.

75. Nong K, Wang W, Niu X, et al. Hepatoprotective effect of exosomes from human-induced pluripotent stem cell-derived mesenchymal stromal cells against hepatic ischemia-reperfusion injury in rats. Cytotherapy 2016;18:1548-59.

76. Ye L, Chang YH, Xiong Q, et al. Cardiac repair in a porcine model of acute myocardial infarction with human induced pluripotent stem cell-derived cardiovascular cells. 


\section{Cell Stem Cell 2014;15:750-61.}

77. Ge X, Wang IN, Toma I, et al. Human amniotic mesenchymal stem cell-derived induced pluripotent stem cells may generate a universal source of cardiac cells. Stem Cells Dev 2012;21:2798-808.

78. Kim PJ, Mahmoudi M, Ge X, et al. Direct evaluation of myocardial viability and stem cell engraftment demonstrates salvage of the injured myocardium. Circ Res 2015;116:e40-50.

79. Lee WH, Chen WY, Shao NY, et al. Comparison of NonCoding RNAs in Exosomes and Functional Efficacy of Human Embryonic Stem Cell- versus Induced Pluripotent Stem Cell-Derived Cardiomyocytes. Stem Cells 2017;35:2138-49.

80. Halkein J, Tabruyn SP, Ricke-Hoch M, et al. MicroRNA- 146a is a therapeutic target and biomarker for peripartum cardiomyopathy. J Clin Invest 2013;123:2143-54.

81. Bang C, Batkai S, Dangwal S, et al. Cardiac fibroblastderived microRNA passenger strand-enriched exosomes mediate cardiomyocyte hypertrophy. J Clin Invest 2014;124:2136-46.

82. Jiang X, Sucharov J, Stauffer BL, et al. Exosomes from pediatric dilated cardiomyopathy patients modulate a pathological response in cardiomyocytes. Am J Physiol Heart Circ Physiol 2017;312:H818-26.

83. Wang X, Huang W, Liu G, et al. Cardiomyocytes mediate anti-angiogenesis in type 2 diabetic rats through the exosomal transfer of miR-320 into endothelial cells. J Mol Cell Cardiol 2014;74:139-50. doi: $10.21037 /$ sci.2017.11.06

Cite this article as: Barile L, Milano G, Vassalli G. Beneficial effects of exosomes secreted by cardiac-derived progenitor cells and other cell types in myocardial ischemia. Stem Cell Investig 2017;4:93. 